\title{
ENTORNO DE COMPETITIVIDAD EN LA REPÚBLICA DOMINICANA
}

\section{DOMINICAN REPUBLIC'S COMPETITIVENESS ENVIRONMENT}

Isidanys Moronta Morel ${ }^{1}$

Víctor Gisbert Soler ${ }^{2}$

1. Ingeniera Industria. Universidad Autónoma de Santo Domingo, República Dominicana. Máster Universitario en Ingeniería en Organización y Logística, Universidad Politécnica de Valencia, (España). E-mail: ismomo@epsa.upv.es

2. Doctor Ingeniero Industrial. Departamento de Estadística e Investigación Operativa Aplicadas y Calidad. Universidad Politécnica de Valencia, (España). E-mail: vgisber@eio.upv.es

\section{Citación sugerida:}

Moronta Morel, I. y Gisbert Soler, V. (2017). Entorno de competitividad en la República Dominicana. 3C Empresa: investigación y pensamiento crítico, Edición Especial, 64-72. DOI: $<$ http://dx.doi.org/10.17993/3cemp.2017.especial.64-72/>. 


\section{RESUMEN}

El presente artículo ha sido realizado en base a estudios e investigaciones previas, el mismo para dar a conocer una visión general de la situación actual de la Republica Dominicana en cuanto a competitividad. Se detallarán los factores más relevantes que definen al país en el aspecto mencionado, cuáles son sus fortalezas, como se posiciona en relación al resto de países latinoamericanos y cuales estrategias han sido planteadas a largo plazo para mejorar.

\section{ABSTRACT}

This article has been written based on previous studies and research, the same to give an overview of the current situation of the Dominican Republic in terms of competitiveness. It will detail the most relevant factors that define the country in the aforementioned aspect, what its strengths are, how it positions itself in relation to the rest of Latin American countries and what strategies have been proposed in the long term to improve.

\section{PALABRAS CLAVE}

República Dominicana, Competitividad, Economía, Desarrollo.

\section{PALABRAS CLAVE}

Dominican Republic, Competitiveness, Economy, Development. 


\section{INTRODUCCIÓN}

República dominicana es un país perteneciente a las Antillas en el continente americano, tiene una superficie de $48,670 \mathrm{Km}^{2}$ y una población estimada de $10,785,240$ habitantes, se ha establecido como una de las economías de mayor crecimiento en América desde hace dos décadas e importantes resultados de han logrado en los últimos años.

La República Dominicana ha registrado un fuerte crecimiento del Producto Interno Bruto (PIB) en 2016, ubicándose nuevamente a la cabeza de la región, alcanzando una tasa de crecimiento de $6.3 \%$ de acuerdo a las últimas proyecciones; si bien este crecimiento se queda ligeramente por debajo del crecimiento del $7.0 \%$ registrado en 2015 , se trata de un resultado muy superior al pronóstico inicial del presupuesto 2016 que se había confeccionado con el objetivo de alcanzar un crecimiento del 5.0\%. Para 2017, se espera que siga frenándose algo más el ritmo de crecimiento, esto como producto de las incertidumbres en el escenario internacional y conflictos internos de la nación.

Las principales fuentes de ingreso y de las cuales depende la Republica Dominicana son la agricultura, el sector comunicaciones, construcción, turismo, zonas francas, remesas y servicios. De todos los sectores los que mayor crecimiento en los últimos tiempos son las zonas francas y el turismo.

Enfrentar con éxito la competencia global implica fortalecer las ventajas competitivas y desarrollar nuevas estrategias a nivel de empresas, clusters, regiones y países. Las nuevas ventajas deberán basar su competitividad en la innovación, el capital intelectual y en la rapidez con la que evolucionan ante el cambio continuo, de tal manera que los lideres serán aquellos que puedan sustentar su ventaja competitiva y tengan la capacidad de aprender, innovar y responder más rápido que la competencia.

A todo esto y tomando en consideración el posicionamiento económico de Republica Dominicana nos preguntamos: ¿Es la Republica Dominicana un país competitivo en relaciona otros países latinoamericanos?

\section{COMPETITIVIDAD. UN ENTORNO GENERAL}

La competitividad es definida por el Foro económico mundial ${ }^{1}$ como "el conjunto de instituciones, políticas y factores que determinan el nivel de productividad de un país".

Michael Porter ${ }^{2}$, el principal exponente de las teorías sobre el tema en todos los niveles y creador del término "Competitividad de las naciones" y lo definió como "La habilidad de los países para crear valor agregado y aumentar el bienestar de la población".

Básicamente, un aumento de la competitividad significa aumento de prosperidad, el Foro económico mundial considera que las economías competitivas son aquellas con más 
probabilidad de crecer de forma sustentable e inclusiva, lo que significa más probabilidad de que todos los miembros de la sociedad se beneficien con los frutos del crecimiento económico.

Basándonos en los conceptos previos debemos entonces establecer la noción de competitividad sistémica, esta es un patrón en el que es estado y los actores de la sociedad civil crean de una forma deliberada las condiciones para un desarrollo industrial exitoso. Este término se refiere a naciones, regiones, sectores industriales o subsectores. Diferenciándose entonces así la competitividad sistémica de la de empresas en que, si una empresa no es competitiva, quiebra, mientras que, si un país o región no lo es, no quiebra, pero experimenta una disminución en el bienestar de la población. Este concepto fue desarrollado por un grupo de investigadores del Instituto alemán de desarrollo.

Entorno a todo esto nos cuestionamos: ¿Cómo podemos cuantificar la competitividad de un país?

Existen factores medibles que pueden ser tomados como punto de referencia para medir la competitividad de un país, estos podemos clasificarlos en tres factores:

1. Subíndice de requisitos básicos. Sustentado por cuatro pilares, este es clave para las economías impulsadas por factores:
a. Instituciones.
b. Infraestructura.
c. Entorno macroeconómico.
d. Salud y educación primaria.

2. Subíndice de potenciadores de eficiencia. Sustentado por seis pilares, este es clave para las economías impulsadas por la eficiencia:
a. Educación superior.
b. Eficiencia del mercado de valores.
c. Eficiencia del mercado laboral.
d. Desarrollo del mercado financiero.
e. Preparación tecnológica.
f. Tamaño del mercado.

3. Subíndice de factores de innovación y sofisticación. Sustentado en dos pilares, este es clave para las economías impulsadas por la innovación:

a. Sofisticación del negocio.

b. Innovación.

Estas son las áreas más complejas de competitividad que requieren una economía que pueda aprovechar negocios de clase mundial y establecimientos de investigación. Aquellos países que presentan mejor valoración y desempeño de la innovación comercial y la sofisticación por lo general son economías avanzadas con producto bruto interno alto per cápita. 


\section{INDICADORES DE COMPETITIVIDAD - REPUBLICA DOMINICANA}

En la nueva economía global los negocios se comportan distinto, se basan y se mueven por el mercado (Market driven), el consumo más personalizado (Mass customizing) y una producción más hecha a la medida (Tailor made), por lo tanto, flexible.

De acuerdo al informe global de competitividad del $2016-2017^{1}$ y analizando los índices básicos, de eficiencia, de innovación y sofisticación, Republica Dominicana ocupa la posición número 92 de 138 países en el ranking de competitividad a nivel mundial con una puntuación de 3.94 sobre 7. En el informe de 2015-2016 ocupaba la posición 98 con una puntuación de 3.86 y en 2015 la 101 con 3.76, lo que nos indica que el país ha realizado cambios positivos para mejorar. Esta medición lo posiciona en el número 14 en relación a los demás países latinoamericanos.

\begin{tabular}{|c|c|c|c|c|c|c|}
\hline \multicolumn{7}{|c|}{ Indice Global de Competitividad. Caso República Dominicana } \\
\hline \multirow{2}{*}{\multicolumn{2}{|c|}{ Indice Global de Competitividad }} & \multicolumn{2}{|c|}{ 2015-2016 } & \multicolumn{2}{|c|}{ 2016-2017 } & \multirow{2}{*}{\begin{tabular}{|c|} 
Variación \\
$16 / 17$ \\
\end{tabular}} \\
\hline & & Posición & Puntaje & Posición & Puntaje & \\
\hline & Índice Global & 98 & 3,86 & 92 & 3,94 & $2,2 \%$ \\
\hline & Requerimientos Básicos & 100 & 4,09 & 95 & 4,22 & $3,1 \%$ \\
\hline Pilar 1 & Instituciones & 118 & 3,27 & 123 & 3,19 & $\cdot 2,6 \%$ \\
\hline Pilar 2 & Infraestructura & 100 & 3,21 & 101 & 3,22 & $0,2 \%$ \\
\hline Pilar 3 & Ambiente macroeconómico & 57 & 4,85 & 26 & 5,54 & $14,2 \%$ \\
\hline Pilar 4 & Salud y educación primaria & 104 & 5,04 & 108 & 4,93 & $-2,2 \%$ \\
\hline \multicolumn{2}{|c|}{ Potenciadores de Eficiencia } & 92 & 3,76 & 92 & 3,83 & $1,7 \%$ \\
\hline Pilar 5 & Educación superior y capacitación & 96 & 3,80 & 95 & 3,94 & $3,7 \%$ \\
\hline Pilar 6 & Eficiencia del mercado de bienes & 97 & 4,09 & 106 & 4,00 & $-2,3 \%$ \\
\hline Pilar 7 & Eficiencia del mercado laboral & 108 & 3,81 & 109 & 3,79 & $-0,5 \%$ \\
\hline Pilar 8 & Desarrollo del mercado financiero & 93 & 3,53 & 87 & 3,73 & $5,6 \%$ \\
\hline Pilar 9 & Disponibilidad tecnológica & 84 & 3,52 & 79 & 3,73 & $5,9 \%$ \\
\hline Pilar 10 & Tamaño del mercado & 70 & 3,83 & 67 & 3,79 & $-1,1 \%$ \\
\hline & Innovación & 97 & 3,36 & 99 & 3,35 & $-0,4 \%$ \\
\hline \begin{tabular}{|l|} 
Pilar 11 \\
\end{tabular} & Sofisticación de los negocios & 76 & 3,81 & 77 & 3,81 & $0,0 \%$ \\
\hline Pilar 12 & Innovación & 112 & 2,92 & 114 & 2,89 & $-1,1 \%$ \\
\hline
\end{tabular}

Tabla 1. Índice global de competitividad. Caso Republica Dominicana. Fuente: Consejo Nacional de competitividad (CNC) ${ }^{3}$. 


\begin{tabular}{|c|c|c|c|c|}
\hline \multicolumn{5}{|c|}{$\begin{array}{l}\text { indice Global de Competitividad, países de América Latina y } \\
\text { el Caribe }\end{array}$} \\
\hline & \multicolumn{2}{|c|}{ 2016-2017 } & \multicolumn{2}{|c|}{ Variación 16/17 } \\
\hline Pais & Ranking & Puntuación & Ranking & Puntuación \\
\hline Chile & 33 & 4,64 & -2 & $1,3 \%$ \\
\hline Panamá & 42 & 4,51 & -8 & $3,0 \%$ \\
\hline México & 51 & 4,41 & -6 & $=2,8 \%$ \\
\hline Costa Rica & 54 & 4,41 & 2 & $1,8 \%$ \\
\hline Colombia & 61 & 4,30 & 0 & $0,5 \%$ \\
\hline Perú & 67 & 4,23 & -2 & $0,5 \%$ \\
\hline Barbados & 72 & 4,19 & & \\
\hline Uruguay & 73 & 4,17 & 0 & $2,0 \%$ \\
\hline Jamaica & 75 & 4,13 & - 11 & - $4,0 \%$ \\
\hline Guatemala & 78 & 4,08 & 0 & $0,7 \%$ \\
\hline Brasil & 81 & 4,06 & 6 & $-0,5 \%$ \\
\hline Honduras & 88 & 3,98 & 00 & $0,8 \%$ \\
\hline Ecuador & 91 & 3,96 & 15 & $-2,7 \%$ \\
\hline República Dominicana & 92 & 3,94 & -6 & $\Delta \quad 2,1 \%$ \\
\hline Trinidad y Tobago & 94 & 3,93 & 5 & $\nabla-0,3 \%$ \\
\hline Nicaragua & 103 & 3,81 & -5 & $1,6 \%$ \\
\hline Argentina & 104 & 3,81 & -2 & $0,5 \%$ \\
\hline El Salvador & 105 & 3,81 & 10 & $-1,6 \%$ \\
\hline Paraguay & 117 & 3,65 & -1 & $1,4 \%$ \\
\hline Bolivia & 121 & 3,54 & 4 & $-1,7 \%$ \\
\hline Venezuela, RB & 130 & 3,27 & -2 & $\nabla_{-0,9 \%}$ \\
\hline
\end{tabular}

Tabla 2. Índice global de competitividad. Países de américa latina y el caribe.

Fuente: Consejo Nacional de competitividad (CNC) ${ }^{3}$.

De acuerdo a los indices de Republica Dominicana podemos decir que el pais caribeño tiene un amplio margen de mejora competitiva y un gran trabajo por hacer, tomando en consideracion la tendencia de los ultimos años se evidencia que se han estado tomando medidas estrategicas para posicionarse mejor en todos los aspectos para ser mas competitivos y tener mayor proyeccion a nivel internacional. Pero, ¿que podemos decir de aquellos factores que retrasan un mejor avance para el mismo y estar mas a la par con paises que ocupan posiciones con mejores indices? Pues, según una encuesta realizada por el foro mundial de la competitividad los problemas principales que frenan el progreso competitivo del pais son los siguientes:

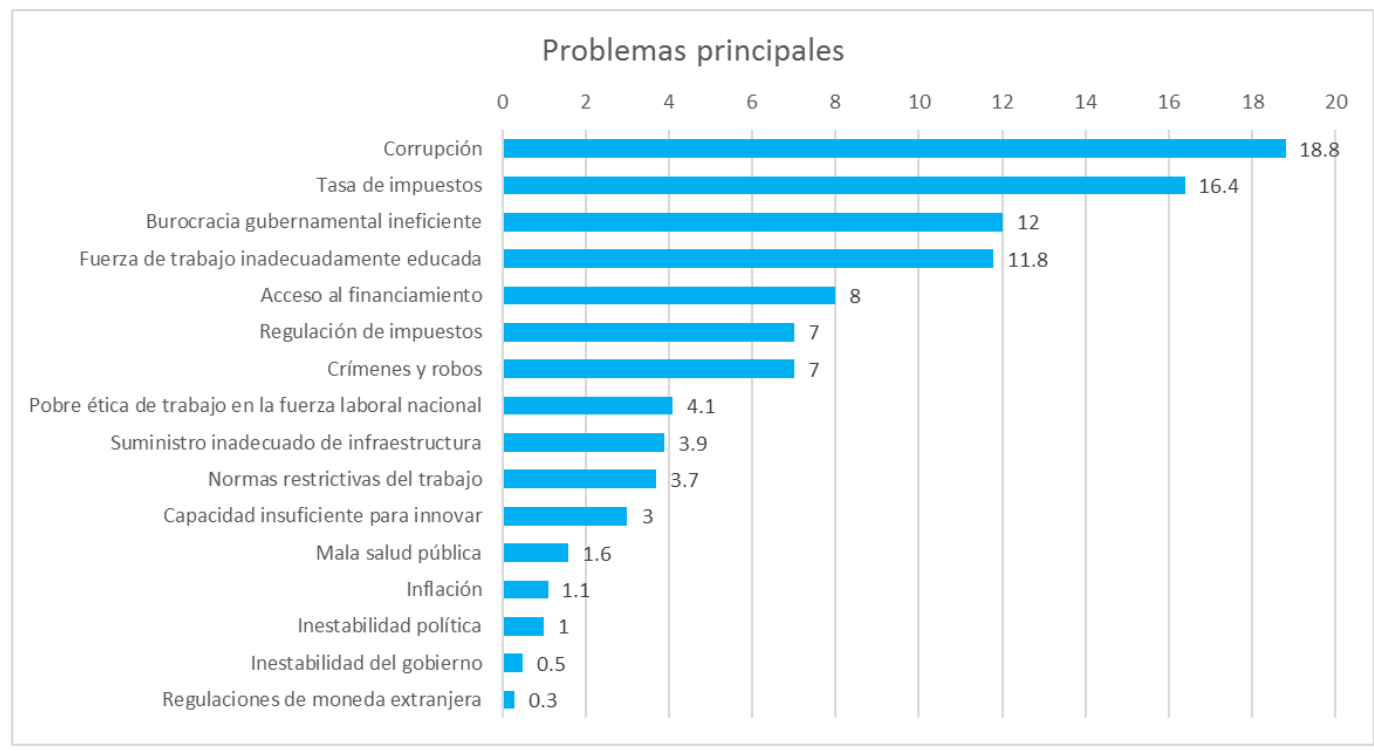

Tabla 3. Principales problemas de Republica Dominicana frente a la competitividad.

Fuente: Consejo Nacional de competitividad $(\mathrm{CNC})^{3}$. 
Además de estos problemas es claramente sabido que la economía dominicana no tiene un problema de crecimiento sino de dirección y estrategia.

Estos factores son un mal común que afecta a mayoría de los países latinoamericanos, sin embargo, Republica Dominicana se encuentra en un proceso de cambio a nivel social y cultural que de manera indirecta está influyendo en reducir estos problemas. Además, a nivel económico han empezado a comprender que el éxito internacional de una nación depende de dos aspectos interrelacionados: el comercio internacional y las inversiones extranjeras directas.

\section{FUTURO COMPETITIVO}

Tras recuperarse en el aspecto macroeconómico, Andrés Vander-Horst ${ }^{5}$ plantea que la Republica dominicana tiene que redefinir un nuevo modelo de desarrollo económico, fortalecer sus instituciones y definir una estrategia de competitividad orientada hacia la producción basada en la innovación y en el uso de tecnologías modernas.

Como uno de los principales problemas que enfrenta la Republica Dominicana en materia de competitividad se resalta la problemática laboral y el desafío de la capacidad para el desarrollo de los trabajadores, del conocimiento, la fragilidad institucional, avanzar en infraestructura logística eficiente y finalmente desarrollar un nuevo enfoque de asociatividad que permita entender que la competencia no es interna sino a nivel global.

El país es parte de tratados de comercio de relevancia, dentro de los que se podrían mencionar el DR-CAFTA, un tratado de libre comercio entre República Dominicana, Centro América y Estados Unidos, también el CARIFORUM un acuerdo de asociación económica entre la Unión Europea y el caribe, estos acuerdos un instrumento de gran utilidad para el desarrollo económico del país, Sin embargo, no son suficiente por si solos para elevar la competitividad sus sectores productivos y garantizar el éxito. Esto dependerá que tan efectivas sean las medidas que tome el país entorno a su competitividad sistémica la participación activa del gobierno, empresas, universidades y sociedad civil en general. El objetivo inmediato de corto plazo del gobierno de Republica Dominicana, ha sido recuperar la estabilidad macroeconómica. Sin embargo, esto es solo la etapa inicial de un proceso complejo que implica la redefinición de un nuevo modelo de desarrollo económico.

Según el plan nacional de competitividad sistémica de la Republica Dominicana, para el 2020 el país estará plenamente integrado a la economía mundial con una plataforma de desarrollo competitiva, sostenible y equitativa. Sin embargo, alcanzar esta visión general implica tener un crecimiento competitivo sustentable que permita generar empleos productivos y salarios remunerativos, para así promover un desarrollo humano integral de los dominicanos, mayor esperanza y calidad de vida, mejor educación y mayor nivel de ingreso per cápita. 
En sentido general Republica dominicana presenta fortalezas que le permiten plantearse una visión de futuro prometedora: a) Es una economía con una capacidad de acumulación de capital y crecimiento de las altas. b) La economía del país presenta una estructura productiva diversificada como fuente de crecimiento. c) Una posición geográfica que le da ventaja comparativa de localización tanto para abastecer a Estados unidos como a Latinoamérica y Europa. El desafío es convertirla en una ventaja competitiva logística.

Hoy día lo único constante es el cambio y lo único cierto es la incertidumbre, el país debe crear planes adaptables, que sean flexibles y vayan cambiando a medida que van cambiando los escenarios en el mundo globalizado en el que vivimos, el gran desafío actual y futuro es pasar del plan a la acción, a sabiendas que la competencia no solo es interna sino global y que los objetivos que el país se ha planteado solo serán alcanzados teniendo una mente abierta, activa y comprometida. 


\section{REFERENCIAS BIBLIOGRÁFICAS}

[1]- Cann, Oliver. Head of Media Content, World Economic Forum Geneva. Foro económico mundial. 2016. https://www.weforum.org/

[2]- Porter, Michael. La ventaja competitiva de las naciones. 1991.

[3]- Consejo Nacional de Competitividad (CNC). División de información estratégica. Octubre 2016.http://www.competitividad.org.do/wpcontent/uploads/2016/10/Informe-Global-de-Competitividad-2016-2017.pdf

[4]- Foro económico mundial. Reporte anual 2016-2017. https://www.weforum.org/reports/the-global-competitiveness-report-2016-2017-1

[5]- Vander-Horst Álvarez, Andrés. Competitividad, desafío global para un reto local. 2006. 